\title{
CORRIGENDUM
}

\section{Nutritional management of children with cerebral palsy: a practical guide}

\author{
MN Kuperminc ${ }^{1}, \mathrm{~F}$ Gottrand ${ }^{2}$, L Samson-Fang ${ }^{3}$, J Arvedson ${ }^{4}, \mathrm{~K}$ Bell ${ }^{5,6}, \mathrm{GM} \mathrm{Craig}^{7}$ and PB Sullivan ${ }^{8}$
}

European Journal of Clinical Nutrition (2014) 68, 284; doi:10.1038/ejcn.2013.281; published online 18 December 2013

Correction to: European Journal of Clinical Nutrition (2013) 67, S21-S23; doi:10.1038/ejen.2013.227

Since the publication of this supplement, it has been noticed that the author affiliations were not included in the Summary and Recommendations article. The correct author affiliations are shown below, and the online html and PDF have been amended.

${ }^{1}$ Division of Neurodevelopmental Pediatrics, Naval Medical Center Portsmouth, Portsmouth, VA, USA; ${ }^{2}$ Hôpital J de Flandre,
CHRU Lille, Lille, France; ${ }^{3}$ University of Utah School of Medicine, Salt Lake City, UT, USA; ${ }^{4}$ Children's Hospital of Wisconsin, Milwaukee, WI, USA; ${ }^{5}$ Queensland Cerebral Palsy and Rehabilitation Research Centre, Children's Nutrition Research Centre, Queensland Children's Medical Research Institute, The University of Queensland, Brisbane, Queensland, Australia; ${ }^{6}$ Department of Paediatric Rehabilitation, Children's Health Queensland Hospital and Health Service, Brisbane, Queensland, Australia; ${ }^{7}$ School of Health Sciences, City University London, London, UK and ${ }^{8}$ Children's Hospital, Oxford University Hospitals NHS Trust, Oxford, UK 